\title{
Correction to: Specific Susceptibility to COVID-19 in Adults with Down Syndrome
}

\author{
Tomer Illouz ${ }^{1,2} \cdot$ Arya Biragyn $^{3} \cdot$ Milana Frenkel-Morgenstern $^{4} \cdot$ Orly Weissberg $^{4} \cdot$ Alessandro Gorohovski $^{4}$. \\ Eugene Merzon ${ }^{5}$. Ilan Green ${ }^{5}$. Florencia lulita ${ }^{6,7,8,9} \cdot$ Lisi Flores-Aguilar $^{10} \cdot$ Mara Dierssen $^{11,12,13}$. \\ Ilario De Toma ${ }^{14,15,16}$. Hefziba Lifshitz ${ }^{17}$. Stylianos E. Antonarakis ${ }^{18,19,20}$. Eugene $\mathrm{Yu}^{21,22}$. Yann Herault ${ }^{23}$. \\ Marie-Claude Potier ${ }^{24}$. Alexandra Botté24 ${ }^{24}$ Randall Roper ${ }^{25}$. Benjamin Sredni ${ }^{26}$. Ronit Sarid ${ }^{24}$. \\ Jacqueline London ${ }^{27} \cdot$ William Mobley ${ }^{28} \cdot$ Andre Strydom $^{29,30} \cdot$ Eitan Okun ${ }^{1,2,26}$ (D)
}

Published online: 19 November 2021

(c) The Author(s) 2021

\section{Correction to: NeuroMolecular Medicine https://doi.org/10.1007/s12017-021-08651-5}

In this article the Acknowledgement section should read as follows:

Acknowledgement This research was supported by the Trisomy-21 Research Society (T21RS). This research was supported in part by the Intramural Research Program of the National Institute on Aging. We thank Yael Laure for thorough editing of the manuscript.

The original article has been corrected.

Publisher's Note Springer Nature remains neutral with regard to jurisdictional claims in published maps and institutional affiliations.
The original article can be found online at https://doi.org/10.1007/ s12017-021-08651-5.

Eitan Okun

eitan.okun@biu.ac.il

1 The Leslie and Susan Gonda Multidisciplinary Brain Research Center, Bar-Ilan University, 5290002 Ramat-Gan, Israel

2 The Paul Feder Laboratory On Alzheimer's Disease Research, Bar-Ilan University, 5290002 Ramat-Gan, Israel

3 Laboratory of Molecular Biology and Immunology, NIA, Baltimore, MD 21224, USA

4 Cancer Genomics and BioComputing of Complex Diseases Lab, Azrieli Faculty of Medicine, Bar-Ilan University, Safed, Israel

5 Leumit Health Services, Department of Family Medicine, Sackler School of Medicine, Tel Aviv University, Tel Aviv-Yafo, Israel

6 Sant Pau Memory Unit, Department of Neurology, Hospital de La Santa Creu I Sant Pau, Barcelona, Spain
7 Biomedical Research Institute Sant Pau, Universitat Autònoma de Barcelona, Barcelona, Spain

8 Center of Biomedical Investigation Network for Neurodegenerative Diseases (CIBERNED), Madrid, Spain

9 Alzheimer-Down Unit, Fundación Catalana Síndrome de Down, Barcelona, Spain

10 Department of Anatomy and Cell Biology, McGill University, Montreal, Canada

11 Center for Genomic Regulation, The Barcelona Institute for Science and Technology, Barcelona, Spain

12 University Pompeu Fabra, Barcelona, Spain

13 Biomedical Research Networking Center for Rare Diseases (CIBERER), Barcelona, Spain

14 Cellular \& Systems Neurobiology, Systems Biology Program, The Barcelona Institute of Science and Technology, Centre for Genomic Regulation (CRG), Dr. Aiguader 88, 08003 Barcelona, Spain

15 Universitat Pompeu Fabra (UPF), Barcelona, Spain 
16 Centro de Investigación Biomédica en Red de Enfermedades Raras (CIBERER), Madrid, Spain

17 School of Education, Bar-Ilan University, 5290002 Ramat-Gan, Israel

18 Department of Genetic Medicine and Development, University of Geneva, 1211 Geneva, Switzerland

19 Medigenome, Swiss Institute of Genomic Medicine, 1207 Geneva, Switzerland

20 iGE3 Institute of Genetics and Genomics of Geneva, 1211 Geneva, Switzerland

21 The Children's Guild Foundation Down Syndrome Research Program, Genetics and Genomics Program and Department of Cancer Genetics and Genomics, Roswell Park Comprehensive Cancer Center, Buffalo, NY, USA

22 Genetics, Genomics and Bioinformatics Program, State University of New York At Buffalo, Buffalo, NY, USA

23 Université de Strasbourg, CNRS, INSERM, Institut de Génétique Biologie Moléculaire Et Cellulaire, IGBMCUMR, 7104 - Inserm U1258, 1 rue Laurent Fries, ILLKIRCH, 67404 Cedex, France
24 Paris Brain Institute (ICM), CNRS UMR7225, INSERM U1127, Sorbonne Université, Hôpital de La Pitié-Salpêtrière, Paris, France

25 Department of Biology, Indiana University-Purdue University Indianapolis, Indianapolis, USA

26 The Mina and Everard Goodman Faculty of Life Sciences, Bar-Ilan University, 5290002 Ramat-Gan, Israel

27 Université de Paris, BFA, UMR 8251, CNRS, 7501 Paris, France

28 Department of Neurosciences, University of California, San Diego, USA

29 Department of Forensic and Neurodevelopmental Sciences, Institute of Psychiatry Psychology and Neuroscience, King's College London, 16 De Crespigny Park, London SE5 8AF, UK

30 South London and Maudsley NHS Foundation Trust, London, UK 